\title{
El control europeo del ciberespacio ante el discurso de odio: análisis de las medidas de lucha y prevención
}

\section{The European Cyberspace Control in Face of Hate Speech: Legal Measures to Combat and Prevent}

\author{
Víctor Luis Gutiérrez Castillo ${ }^{1}$ \\ Universidad de Jaén (España)
}

Recibido: 01-09-20

Aceptado: 02-10-20

\section{Resumen}

El recurso a internet como medio de difusión del hate speech plantea una serie de interrogantes a los que hay que dar una respuesta desde el derecho: ¿donde se encuentra el límite de la libertad de expresión?, ¿deben responder los servidores de internet por los contenidos publicados? ¿qué medidas se están adoptando en Europa? En este trabajo proponemos hacer un análisis crítico de las medidas de control existentes en Europa para prevenir y luchar contra el discurso de odio en el ciberespacio. Se prestará una especial atención a la posible responsabilidad de los portales de internet, así como al código de conducta seguido en el marco de la Unión europea.

Palabras-clave: Libertad de expresión, internet, discurso de odio, Europa, autorregulación.

\begin{abstract}
The use of the Internet as a means of disseminating hate speech raises a series of questions to which an answer must be given from the law: where is the limit of freedom of expression? Should internet service providers be liable for published

\footnotetext{
${ }^{1}$ (vlguti@ujaen.es). Profesor Titular de Derecho Internacional Público y Relaciones Internacionales de la Universidad de Jaén. Entre sus publicaciones cabe mencionar las siguientes: España y sus fronteras en el mar, Dykinson, Madrid, 2004; "Estados árabes y Derechos humanos: la recepción y aplicación de la norma internacional”, REDI, vol. LXIX, 2012, pp. 105-131; El desarrollo y consolidación del espacio de libertad, seguridad y justicia de la Unión Europea, Tecnos, Madrid, 2016.

ORCID: https://orcid.org/0000-0002-9329-2836.
} 
content? What measures are being taken in Europe? In this paper, we propose to make a critical analysis of the existing control measures in Europe to prevent and combat hate speech in cyberspace. Special attention will be paid to the possible liability of internet portals, as well as to the code of conduct followed within the framework of the European Union.

Key-words: Freedom of Expression, Internet, Hate Speech, Europe, SelfRegulation.

\section{Algunas consideraciones históricas sobre la protección de la liber- tad de expresión y opinión}

La libertad de expresión es uno de los derechos más antiguos protegidos por los sistemas jurídicos nacionales. De hecho, en los documentos constitucionales que se desarrollaron durante el siglo XVIII ya se establecía la necesidad de garantizar dicha libertad. Piénsese, por ejemplo, en la Declaración de los Derechos del Hombre y del Ciudadano, de 26 de agosto de 1789, cuyo artículo 11 reconoce el derecho de cualquier ciudadano a "... hablar, escribir e imprimir libremente, siempre y cuando responda del abuso de esta libertad en los casos determinados por la Ley"2. Libertad ésta, que años antes, ya había sido reconocida en el continente americano, con ocasión de la proclamación de la Declaración de Virginia de 12 de junio de 1776. Desde entonces, y hasta nuestros días, todas las constituciones de Estados libres han aceptado su protección como garantía individual. En este orden de ideas, cabe recordar el famoso discurso de las Cuatro Libertades (Four Freedoms Speech) formulado por el presidente Roosevelt ante el Congreso de Estados Unidos en 1941, en el que se reconoce como ejes fundamentales para el futuro de la humanidad el disfrute de la "libertad de culto", la "libertad de vivir sin penuria", la "libertad de vivir sin miedo" y en primera instancia: "la libertad de palabra y expresión"3. Planteamiento éste que, sin duda, influyó en la redacción de textos internacionales venideros, como la Declaración Universal de los Derechos Humanos -DUDH- y el Pacto Internacional de Derechos Civiles y Políticos (PIDCP), cuyo artículo 20, además, apela a la prohibición por ley de toda apología del odio que constituya incitación a la discriminación, la hostilidad o la violencia. A la protección de estos textos se sumaría con el tiempo, la proporcionada por otros instrumentos internacionales específicos, como

\footnotetext{
2 Pascal Nicollier, La Déclaration des Droits de l'Homme et Citoyen du 26 août 1789, Fribourg, 1995.

3 Bryan-Paul Frost, Jeffrey Sikkenga, History of American Political Thought, Maryland, Lexington, 2019.
} 
la Convención de Naciones Unidas para la eliminación de todas las formas de discriminación racial de 1965, así como los instrumentos regionales de protección de derechos humanos, entre los que destacan el Convenio Europeo para la Protección de los Derechos Humanos y de las Libertades Fundamentales (CEDH); la Convención Americana sobre Derechos Humanos, la Convención Africana sobre Derechos Humanos y de los Pueblos y la Declaración de la Asociación de Naciones del Sureste Asiático.

Ahora bien, todos estos textos internacionales, además de proteger la libertad de expresión, prevén la posibilidad de limitarla o restringirla al objeto de evitar lesiones a terceros. En este contexto el discurso de odio constituye una de las causas por las que se puede (y debe) limitar la libertad de expresión, dado su potencial lesivo contra los derechos de grupos vulnerables de los individuos que los conforman. En este orden de ideas, cobra sentido las teorías de Gordon Allport, quien en 1954 elaboró la escala Allport del prejuicio y la discriminación, con la intención de medir los diferentes grados de violencia en una sociedad ${ }^{4}$. Dicha escala se articula en una secuencia de cinco grados de odio, de menor a mayor. El discurso de odio estaría en primer estadio: antilocución o antagonismo verbal, es decir, cuando un grupo de personas muestra libremente una imagen negativa de otro grupo de personas. Aunque el antagonismo verbal no constituye en sí mismo una lesión, puede preparar el escenario para desenlaces violentos, de ahí la necesidad de limitar la libertad en estos casos. Los siguientes estadios de odio, según Allport serían la evitación (cuando se evita activamente a los miembros de un grupo, pudiendo ocasionar daño psicológico a través de la exclusión social), la discriminación (cuando los prejuicios se convierten en acciones y los individuos de un grupo comienzan a ser discriminados a través de la negación de oportunidades y servicios), el ataque físico (cuando un grupo vandaliza, quema o destruye la propiedad de otro grupo y lleva a cabo actos de violencia) y, finalmente, el exterminio, cuando un grupo de personas busca la exterminación o remoción de otro grupo de personas.

Las últimas décadas del siglo XX se han caracterizado por una profunda transformación de la sociedad: el desarrollo y la difusión exponencial de las tecnologías de la comunicación ha influido en diferentes aspectos de la vida social, económica, política y cultural, redefiniendo los conceptos de tiempo, espacio e identidad. De hecho, la presencia de las nuevas tecnologías, y en especial internet en nuestras vidas, ha provocado una serie de cambios en la manera de comunicar y distribuir la información. En este orden de ideas, el Relator de las Naciones Unidas sobre libertad de expresión publicó en mayo del 2011 un informe sobre la promoción y protección del derecho a la libertad de

${ }^{4}$ Gordon W. Allport, The nature of prejudice, Addison-Wesley Publishing company, London, 1954. 
opinión y expresión ${ }^{5}$ en el que reconoce expresamente el efecto revolucionario de internet en las nuevas tecnologías de la información. Afirmando además que este medio interactivo facilita el intercambio participativo de información, convirtiéndose en un instrumento fundamental para que el ejercido de la libertad de expresión en nuestros días.

Gracias a esta revolución, los contenidos en el ciberespacio circulan libremente, sin que su control sea exclusivo ni centralizado, constituyendo un nuevo reto para la protección de los individuos al que hay que dar respuesta. De hecho, en la década de los noventa, ya tuvieron lugar los primeros estudios en EE.UU. sobre el problema del hate speech online, los derechos civiles en el ciberespacio y los nuevos desafíos de la sociedad digital. El nuevo medio de comunicación e información, suponía un avance y una conquista: hacía posible la comunicación entre sus usuarios en tiempo real y a bajo coste. Sin embargo, su uso también exigía soluciones para evitar la incitación a la violencia en la red, en un espacio en el que el tradicional concepto de jurisdicción estaba destinado a desaparecer.

\section{Lucha y prevención contra el discurso de incitación al odio: marco jurídico internacional}

El hate speech, tradicionalmente traducido como "discurso de odio" es una categoría de la jurisprudencia estadounidense que desde hace años forma parte de la europea, entendiéndose por tal aquel discurso que tiene por objetivo expresar odio e intolerancia hacia una persona o grupo de personas. Podría decirse que, desde el punto de vista internacional, no existe un consenso ni una noción compartida, sobre lo que debe entenderse por tal. De hecho, su percepción, depende de las diferentes sensibilidades jurídicas (sistema continental europeo o anglosajón) y de las políticas existentes en una sociedad. Así, por ejemplo, en sectores de lucha contra el racismo, se considera hate speech el discurso que busca la discriminación o perjuicio a una minoría étnica; para los intelectuales del feminismo, lo es la pornografía, mientras que para la comunidad judía lo son las teorías o discursos negacionistas.

El primer convenio internacional en regular de forma explícita y desarrollada la prohibición del "discurso de odio" ha sido el Convenio Internacional sobre la Eliminación de todas las Formas de Discriminación Racial de 21 de diciembre de 1965 (ICERD, en sus siglas en inglés), en virtud del cual (artículo 4) los Estados parte

\footnotetext{
${ }^{5}$ Informe del Relator Especial sobre la promoción y protección del derecho a la libertad de opinión y de expresión, Frank La Rue. Naciones Unidas. A/HRC/17/27, 16 de mayo de 2011 https://www. acnur.org/fileadmin/Documentos/BDL/2015/10048.pdf?view=1
} 
(...) condenan toda propaganda y todas las organizaciones que se inspiren en ideas o teorías basadas en la superioridad de una raza o de un grupo de personas de un determinado color $\mathrm{u}$ origen étnico, o que pretendan justificar o promover el odio racial y la discriminación racial, cualquiera que sea su forma.

Siguiendo este planteamiento el Comité para la Eliminación de la Discriminación Racial ha emitido varias recomendaciones con las que ha contribuido a una mayor comprensión e interpretación de esta norma internacional como, por ejemplo, las Recomendaciones $\mathrm{n}^{\circ} .7, \mathrm{n}^{\circ} .15$ y la $\mathrm{n}^{\circ}$. 35, en las que subraya el deber de los Estados, no solo de promulgar las leyes pertinentes para la lucha y prevención contra la incitación al odio, sino también la de garantizar su eficaz aplicación ${ }^{6}$. Otras disposiciones claves son las contenidas en el ya citado PIDCP, cuyo artículo 19 prevé que toda persona tiene derecho a la libertad de expresión; entendiendo por ésta la libertad de buscar, recibir y difundir informaciones e ideas de toda índole, sin consideración de fronteras, ya sea oralmente, por escrito, en forma impresa o artística, o por cualquier otro procedimiento de su elección. Ahora bien, el apartado tercero de este artículo, es el que fija los motivos que habilitan a su restricción. En concreto, considera que su ejercicio entraña deberes y responsabilidades especiales, pudiendo quedar sujetas a restricciones que deberán estar expresamente fijadas por ley, siendo necesarias para asegurar a) el respeto a los derechos o a la reputación de los demás y b) la protección de la seguridad nacional, el orden público, la salud o la moral publica. Por el contrario, el artículo 20, como ya vimos, prevé una prohibición total de dicha libertad, que no será modulable en ningún caso. Estas disposiciones, junto con las contenidas en los apartados $3 \mathrm{c}$ ) de la Convención para la Prevención y la Sanción del delito de Genocidio de $1948^{7}$ y el artículo 4 del ICERD, constituyen, según el Relator sobre la libertad de expresión de Naciones Unidas de 2011, el cuerpo legal internacional de delimitación de la prohibición del "discurso de incitación al odio" 8 .

Paralelamente a los convenios internacionales, no se puede obviar la importancia de la jurisprudencia internacional y el soft law en la determinación de limites a la libertad de expresión ante el discurso de odio. Entre el conjunto de reglamentaciones soft law, cabe destacar el Plan de Acción de Rabat sobre la prohibición de la apología del odio nacional, racial o religioso, que

\footnotetext{
${ }^{6}$ En concreto esta referencia la encontramos en la Recomendación General $\mathrm{n}^{\circ} .15$ relativa al artículo 4 de la Convención ( $42^{\circ}$ periodo de sesiones, 1993). CERD Recomendación General, apartado 2. https://www.hchr.org.co/documentoseinformes/documentos/html/informes/onu/cedr/ recomendacion_15_convencion_discr_racial.html

${ }^{7}$ Adoptada y abierta a la firma y ratificación, o adhesión, por la Asamblea General en su Resolución 260 A (III), de 9 de diciembre de 1948 https://www.ohchr.org/SP/ProfessionalInterest/ Pages/CrimeOfGenocide.aspx

${ }^{8}$ Véase informe "Promoción y protección del derecho a la libertad de opinión y de expresión". AGNU, A/HRC/17/27, 16 de mayo del 2011. Puede consultarse en https://www.acnur.org/fileadmin/ Documentos/BDL/2015/10048.pdf
} 
constituye incitación a la discriminación, la hostilidad o la violencia aprobado en 2012 y que ha influido en numerosas políticas nacionales. Dicho plan es una iniciativa de la Oficina del Alto Comisionado para los Derechos Humanos, que promovió un conjunto de talleres sobre la prohibición de la incitación al odio nacional, racial o religioso, en los que se examinaron pautas legislativas, prácticas judiciales y políticas en esta materia. Dicho plan (del que se hace eco el Alto Comisionado de las Naciones Unidas para los Derechos Humanos, en su informe de 11 de enero del 2013) parte del siguiente razonamiento: las respuestas legales son importantes, pero cualquier legislación debe complementarse con iniciativas de diversos sectores sociales que alimenten la consciencia y el debate social ${ }^{9}$. Por último, en relación a la jurisprudencia internacional no podemos obviar los pronunciamientos del Tribunal Especial para Ruanda, en los casos Nahimena, Barayaguriza y Ngeze, en los que se condenó a titulares de medios de comunicación social por emitir discursos de odio que alentaban a la violencia contra una parte de la población, promoviendo así el genocidio ${ }^{10}$.

\section{Lucha y prevención contra el discurso de incitación al odio en Europa}

Por lo que respecta al continente europeo, encontramos una interesante y extensa normativa regional tanto en el marco del Consejo de Europa como de la UE con relación a la regulación de la libertad de expresión y su posible limitación. Como veremos a continuación, en continente existe una gran sensibilidad ante la problemática del discurso de incitación al odio, por lo que, en la práctica, se tolera y acepta una amplia restricción de la libertad de expresión con el fin de proteger los derechos de terceros. Circunstancia ésta que contrasta con lo que viene sucediendo en EEUU: la perspectiva jurídica americana, fundada sobre la base de la primera enmienda de la Constitución, no acepta injerencia alguna de los poderes públicos en el ejercicio de la libertad de expresión, por lo que no se prevé la posibilidad de limitaciones en relación con los contenidos expresados. De hecho, en EEUU los mensajes más discutibles, impopulares e incluso escabrosos, pueden ser difundidos libremente en lo que se conoce como free Marketplace of ideas. Por esta razón, cualquier competencia dada a los intermediarios digitales, en especial el recurso a técnicas de filtrado preventivo

\footnotetext{
${ }^{9}$ Informe de la Alto Comisionado de las Naciones Unidas para los Derechos Humanos acerca de los talleres de expertos sobre la prohibición de la incitación al odio nacional, racial o religioso. Asamblea General de Naciones Unidas, A/HRC/22/17/Add.4. Disponible en https://www.ohchr.org/ Documents/HRBodies/HRCouncil/RegularSession/Session22/A-HRC-22-17-Add4_sp.pdf

10 Para más información sobre el análisis de este caso véase Daniel Rodríguez Vázquez, "El genocidio de Ruanda: análisis de los factores que influyeron en el conflicto", en Instituto Español de Estudios Estratégicos, Documento de opinión 59 (2017), disponible en http://www.ieee.es/Galerias/ fichero/docs_opinion/2017/DIEEEO59-2017_Genocidio_Ruanda_DanielRguezVazquez.pdf
} 
de contenidos, es percibida por el operador jurídico con recelo, entendiendo que puede dar lugar a una forma de collateral censorship (expresión utilizada por J. M. Balkin ${ }^{11}$ ), incompatible con el precepto constitucional.

Llama la atención el hecho de que el CEDH, a diferencia del PIDCP, no contiene ninguna provisión específica sobre el discurso de odio, limitándose a reconocer posibles restricciones a la libertad de expresión (art. 10.2) y excluyendo aquellas actividades o actos tendentes a lesionar derechos y libertades de terceros. En concreto, las limitaciones a la libertad de expresión que contempla el Convenio, deberán estar dirigidas a proteger la seguridad nacional, la integridad territorial o la seguridad pública, así como la defensa del orden, la protección de la salud, la moral y la prevención del delito. La protección del individuo ante un abuso de libertad de expresión, conecta con la prohibición de discriminación que contempla el Convenio en su artículo 14 al afirmar que el goce de los derechos y libertades reconocidos se asegurará sin distinción alguna, especialmente por razones de sexo, raza, color, lengua, religión, opiniones políticas, origen nacional o social, pertenencia a una minoría nacional, fortuna, nacimiento o cualquier otra situación. Esta previsión también la encontramos en el artículo 1 del Protocolo ${ }^{\circ}$. 12 de 4 de noviembre del 2000.

Con una finalidad interpretativa, la Comisión Europea contra el Racismo y la Intolerancia del Consejo de Europa, en su Recomendación 20 del Comité de Ministros, de 30 de octubre de 1997, hizo uso de una definición, que sería muy bien acogida en el ámbito académico ${ }^{12}$, si bien más recientemente, ha proporcionado una más completa en su Recomendación de Política General n. ${ }^{\circ}$ 15 , de 8 de diciembre de $2015^{13}$. En los considerandos de este texto se parte de una definición amplia, entendiendo por discurso de odio

...el fomento, promoción o instigación, en cualquiera de sus formas, del odio, la humillación o el menosprecio de una persona o grupo de personas, así como el acoso, descrédito, difusión de estereotipos negativos, estigmatización o amenaza con respecto a dicha persona o grupo de personas y la justificación de esas manifestaciones por razones de raza, color, ascendencia, origen nacional o étnico, edad, discapacidad, lengua, religión o creencias, sexo, género, identidad de género, orientación sexual y otras características o condición personales.

${ }^{11}$ Jack M. Balkin, "Old-school/new-school speech regulation", en Harvard Law Review, 127 (2014), pp. 2296- 2342.

${ }^{12}$ La Recomendación $\mathrm{n}^{\circ} .20$ del Comité de Ministros, de 30 de octubre de 1997, definió la incitación al odio como «todas las formas de expresión que propagan, incitan, promueven o justifican el odio racial, la xenofobia, el antisemitismo y otras formas de odio basadas en la intolerancia, entre otras, la intolerancia expresada por el nacionalismo agresivo y el etnocentrismo, la discriminación y la hostilidad contra las minorías, los inmigrantes y las personas de origen inmigrante».

${ }^{13}$ ECRI General Policy Recommendation $\mathrm{n}^{\circ} .15$ on combating hate speech, de 8 de diciembre de 2015.

Araucaria. Revista Iberoamericana de Filosofí, Política, Humanidades y Relaciones Internacionales, año $22, \mathrm{n}^{\circ} 45$. Tercer cuatrimestre de 2020. Pp. 291-310. ISSN 1575-6823 e-ISSN 2340-2199 https://dx.doi.org/10.12795/araucaria.2020.i45.12 
Sin duda, esta definición, más conceptual que jurídica, es la de mayor acogida entre las instituciones europeas y nacionales. En ella, se engloban dos tipos de conductas: por un lado, las propiamente instigadoras de odio y discriminación y, por otro lado, las que denigran a las personas o grupos de personas. Con esta Recomendación, además, el Comité interpela a los Estados miembros a establecer un marco jurídico sólido, que contenga disposiciones sancionadoras contra el discurso de odio. Planteamiento éste que ha sido secundado por la Comisión Europea contra el Racismo y la Intolerancia (ECRI), a través de su Recomendación de política general $\mathrm{n}^{\circ}$. 7, de 13 de diciembre del 2002, sobre legislación nacional para luchar contra el racismo y discriminación racial ${ }^{14}$.

También en el seno del Consejo de Europa, conviene recordar el Protocolo Adicional al Convenio sobre la Ciberdelincuencia (más comúnmente conocida como Convención de Budapest del 2001 ${ }^{15}$ ), relativo a la penalización de actos de índole racista y xenófoba cometidos por sistemas informáticos, hecho en Estrasburgo el 28 de enero de $2003^{16}$, por el que los Estados se comprometen a tipificar como delito la difusión de material racista y xenófobo por medio de un sistema informático, así como las amenazas y la difusión de material negacionista de crímenes contra la humanidad. De esta forma, como veremos en siguientes apartados, se amplia el campo de sanción de la conducta más allá de la difusión de material incitador, abarcando también aquel material que "propugne" o "promueva" el odio, la discriminación o la violencia.

Por lo que se refiere a la jurisprudencia del TEDH, cabe señalar que el tribunal ha tenido la oportunidad de pronunciarse en varias ocasiones sobre los límites de la libertad de expresión ante el discurso de odio. De hecho, lo mencionó expresamente por primera vez en 1999 en cuatro casos contra Turquía ${ }^{17}$, equiparándolo a la "glorificación" o "promoción de la violencia". Sin embargo, nunca ha dado una definición precisa de lo que debe entenderse por tal, remitiéndose a la definición de la ya mencionada Recomendación $\mathrm{n}^{\circ}$. 20, de 30 de octubre de 1997 del Comité de Ministros del Consejo de Europa ${ }^{18}$. Lo que sí ha hecho el tribunal ha sido reconocer explícitamente en su doctrina la existencia de dos grandes categorías de odio: la incitación y la denigración.

14 CRI (2003) 8 REV. Puede consultarse en https://rm.coe.int/ecri-general-policy...no$7 . . .16808 \mathrm{~b} 5 \mathrm{aaf}$

${ }_{15}$ Convenio sobre cibercrimen, abierto a firma el 23 de noviembre del 2001 y en vigor desde el 1 de julio del 2004. Puede consultarse la ficha de este convenio en la página https://www.coe.int/en/web/ conventions/full-list/-/conventions/treaty/185

${ }^{16} \mathrm{BOE} \mathrm{n}^{\circ} .26$, de 30 de enero de 2015.

17 SSTEDH de 8 de julio de 1999 c. Turquía: Sürek (n.o 1), apdo. 62; Sürek y Özdemir, apdo. 63; Sürek (n.o 4), apdo. 60, y Erdogdu e Ince, apdo. 54

${ }^{18}$ Göran Rollnert Liern, "El discurso del odio: una lectura crítica de la regulación internacional", en Revista Española de Derecho Constitucional, 115 (2019), p. 91. 
Ahora bien, por lo que se a la restricción a la libertad de expresión se refiere, el TEDH, al igual que otros organismos internacionales, ha seguido un sistema de evaluación acumulativa: el denominado "test de Estrasburgo" por el que se exige que toda restricción debe estar prevista por ley y obedecer a un propósito legitimo y proporcionado. Además de estas exigencias, la jurisprudencia del tribunal parece haber tenido en cuenta en la práctica otra circunstancia, la conocida como "pressing social need": un balance de coste-beneficio colectivo, en el que el tribunal valora la justificación de la limitación en términos de consecución democrática o de promoción de derechos fundamentales.

Teniendo en cuenta lo expuesto, puede afirmarse que, en el ámbito del Consejo de Europa, el estándar del discurso de odio punible se aplica a comportamientos que no llegan a constituir la incitación, como por ejemplo la propagación, promoción o, incluso, la justificación del odio. Acciones éstas que no suponen en cuanto tal una apelación directa a la comisión de una acción violenta o discriminatoria. Sin embargo, a partir de lo expuesto, podría decirse que para el tribunal la justificación de la violencia es equiparable a la incitación, mientras que su "glorificación" o "promoción" lo es al discurso de odio. Para los jueces de Estrasburgo los ataques que se cometen al injuriar, ridiculizar o difamar a grupos vulnerables o a los individuos que los conforman pueden ser suficientes para limitar la libertad de expresión ${ }^{19}$. Y es que, como ya advertimos, existe una profunda diferencia entre la protección de esta libertad en Europa y en EEUU.

Todo esto explica que en el continente europeo se haya considerado el negacionismo del Holocausto como "una de las más graves formas de difamación racial de los judíos y de incitación al odio contra los mismos"20. Podría afirmarse, pues, que en Europa el umbral de la limitación de la libertad de expresión e incluso la sanción contra el discurso del odio se sitúa debajo de "la incitación indirecta". Piénsese, por ejemplo, en el asunto Vejdeland y otros c. Suecia, de 9 febrero de 2012, en el que el TEDH avaló la sanción impuesta por los tribunales suecos a un sindicato estudiantil que había distribuido panfletos contrarios a la homosexualidad en un colegio. Para el tribunal dicha sanción no violaba la libertad de expresión "a pesar de que estas declaraciones no incitaban directamente a las personas a cometer actos de odio" 21 . Sin embargo, para el tribunal había que tener en cuenta la naturaleza del público receptor de los mensajes (sensible por su juventud) y el carácter denigrante de los mismos contra un colectivo especialmente vulnerable. Siguiendo esta línea argumental, el tribunal también ha considerado justificada la sanción penal de discursos que

\footnotetext{
${ }^{19}$ La sentencia más representativa de esta tendencia es la pronunciada en el asunto Féret c. Bélgica, de 16 de julio de 2009.

${ }^{20}$ Decisión de inadmisibilidad Garaudy c. Francia, de 24 de junio de 2003, apdo. 1. i) de los fundamentos de derecho.

${ }^{21}$ STEDH Vejdeland y otros c. Suecia, de 9 febrero de 2012, apdo. 54.
} 
pueden suscitar "rechazo", "hostilidad" u "odio" hacia un grupo en concreto 22 , considerando este tipo de discurso incompatible con los valores del Convenio ${ }^{23}$.

Por lo que respecta al ámbito comunitario, la UE también ha desplegado una política para combatir y contrarrestar el hate speech, adoptando diversas medidas por las que se incita a los Estados miembros a realizar acciones concretas. Todas ellas toman como fundamento el principio de no discriminación, protegido en el artículo 13 del Tratado de Ámsterdam ${ }^{24}$ y el artículo 21 de la Carta de los Derechos Fundamentales de la UE (en adelante, CDFUE), del 18 de diciembre del $2000^{25}$, que amplía el catálogo de ejes de discriminación, siendo desarrollado por sendas directivas. En concreto, la Directiva 2000/43/ CE del Consejo, de 29 de junio de 2000, relativa a la aplicación del principio de igualdad de trato de las personas independientemente de su origen racial o étnico, tanto en el ámbito privado como público ${ }^{26}$; la Directiva 2000/78/CE del Consejo, de 27 de noviembre de 2000, relativa al establecimiento de un marco general para la igualdad de trato en el empleo y la ocupación ${ }^{27}$, así como la Directiva 2006/54/CE del Parlamento Europeo y del Consejo, de 5 de julio del 2006, relativa a la aplicación del principio de igualdad de oportunidades e igualdad de trato entre hombres y mujeres en asuntos de empleo y ocupación ${ }^{28}$. En conclusión, tales disposiciones ofrecen a un cuadro normativo efectivo, a partir del cual los Estados pueden participar activamente en la lucha contra la discriminación ${ }^{29}$.

Ahora bien, un instrumento clave en esta materia ha sido la Decisión Marco 2008/913/JAI del Consejo de la UE, de 28 de noviembre de 2008, relativa a la lucha contra determinadas formas y manifestaciones de racismo y xenofobia mediante el derecho penal ${ }^{30}$, que significó un paso fundamental en el reconocimiento de los delitos de odio en el ámbito europeo al establecer un objetivo común en la respuesta ante este fenómeno. Tal decisión apela a los Estados a adoptar las medidas que sean necesarias para garantizar la sanción de las conductas relativas a la incitación al odio dirigida contra un grupo de personas (o alguno/os de sus miembros), así como la sanción de la apología, la

\footnotetext{
${ }^{22}$ Féret, apdo. 69, y Decisión de inadmisibilidad Le Pen c. Francia, de 20 de abril de 2010, apdo. 1 de los fundamentos de derecho.

${ }^{23}$ Decisión de inadmisibilidad Pavel Ivanov c. Rusia, de 20 de febrero de 2007, apdo. 1 de los fundamentos de derecho.

${ }^{24}$ El principio de no discriminación forma parte de los principios fundamentales de la UE y ha obtenido el reconocimiento formal con el Tratado de Ámsterdam, por el que se ha modificado el artículo 13 del Tratado sobre la Comunidad Europea, hoy art 19 del TFUE. Versiones consolidadas del Tratado de la UE y del Tratado de Funcionamiento de la UE - Protocolos - Anexos - Diario Oficial $n^{\circ}$. C 326 de 26/10/2012.

${ }_{25}$ Diario Oficial no. C 364 de 18/12/2000.

${ }^{26}$ Diario Oficial n ${ }^{\circ}$. L 180 de 19/07/2000.

${ }^{27}$ Diario Oficial $n^{\circ}$. L 303 de 02/12/2000.

28 Diario Oficial n. L 204 de 26/07/2006.

${ }^{29}$ Maurizio Menzi/Pietro Falleta, Il diritto del Web. Casi e materiali, CEDAM, Trento, 2015, p.176.

${ }^{30}$ Diario Oficial n ${ }^{\circ}$. L 328 de 6/12/2008.
} 
negación o la trivialización flagrante de los crímenes de genocidio, crímenes contra la humanidad y crímenes de guerra. Esta decisión, aunque supone un avance en la sensibilización y lucha contra el discurso de odio, es incompleta, ya que no contempla la discriminación por razón de orientación sexual o razones religiosas entre las categorías susceptibles de incitación al odio. Razón por la que, el Parlamento europeo, con una Resolución aprobada el 14 marzo 2013 sobre el refuerzo de la lucha contra el racismo, la xenofobia y los delitos motivados por el odio, puso de manifiesto la necesidad de revisarla con la intención de incluir la protección ante los discursos homófobos y tránsfobos ${ }^{31}$.

\section{El discurso de incitación al odio on line en el ciberespacio europeo}

\subsection{La culpa in vigilando de los portales de internet: análisis a la luz de la jurisprudencia europea}

El Consejo de Europea ha demostrado una particular atención por la lucha y prevención de la discriminación, así como la incitación al odio en la red, como muestra el hecho de haber adoptado dos acuerdos internacionales que constituyen instrumentos de gran utilidad ante los crímenes informáticos, específicamente en lo relativo a los comportamientos discriminatorios. En primer lugar, el Convenio de Budapest sobre ciberdelincuencia ${ }^{32} \mathrm{y}$, en un segundo lugar, su Protocolo adicional, que ya mencionamos, relativo a la incriminación de actos de naturaleza racista y xenofóbica a través de sistemas informáticos ${ }^{33}$, por el que se fijan dos objetivos principales: concretar el derecho penal en esta materia y mejorar la cooperación internacional entre los Estados ${ }^{34}$. Asimismo, en este ámbito también destacan un conjunto de medidas comprendidas en la categoría de soft law, como la Recomendación de Política General $n^{\circ} .6$ de la ECRI sobre la lucha contra la difusión de materiales racistas, xenófobas y antisemitas en internet de 15 de diciembre de 2000 (por la que se apela a los Estados a integrar la batalla contra estas tendencias en sus políticas de control de contenidos ilícitos en Internet) y la Resolución n". 2144 "Ending cyberdiscrimination and online hate" de la Asamblea Parlamentaria del Consejo de Europa de 2017, por la que se reclama la necesidad de clarificar la responsabilidad de las plataformas intermediarias ${ }^{35}$.

${ }^{31}$ Diario Oficial C 36/81, de 29/01/2016.

${ }^{32}$ Véase nota 18.

${ }^{33}$ El 1 de marzo de 2006 entró en vigor el Protocolo Adicional a la Convención sobre el delito cibernético. ETS n $\mathrm{n}^{\circ} 189$ Additional Protocol to the Convention on Cybercrime, concerning the criminalisation of acts of a racist and xenophobic nature committed through computer systems, disponible en https://www.coe.int/en/web/conventions/full-list/-/conventions/treaty/189/signatures

${ }^{34}$ Para una interesante reflexión jurídica sobre la necesidad de penalizar el discurso de odio, véase Charles Girard, ¿"Pourquoi punir les discours de haine?”, Esprit, 10 (2015), pp. 11-22.

${ }_{35}$ El texto puede consultarse en http://www.assembly.coe.int/nw/xml/XRef/Xref-XML2HTML- 
Por lo que a la libertad de expresión se refiere, el TEDH ha reiterado expresamente, desde la sentencia Handyside c. Reino Unido de 1976, que esta expresión "...constituye uno de los fundamentos esenciales de una sociedad democrática y una de las condiciones primordiales de su progreso"36, reconociendo en varias ocasiones (Times Newspaper Ltd c. Reino Unido de 2009 y Delfi S.A. c. Estonia de 2015) que los principios generales de dicha libertad también son plenamente aplicables a la red $^{37}$. Asimismo, en cuanto a las restricciones a este derecho en el entorno digital, el tribunal ha sido contundente a la hora de considerar incompatible con la libertad de expresión el bloqueo de acceso a internet (asunto Ahmet Yilidirim c. Turquía de 2012 ${ }^{38}$ ); contundencia que, sin embargo, no parece haber manifestado a la hora de determinar responsabilidades ante la circulación de mensajes y discursos que incitan al odio en el ciberespacio. De hecho, han sido solo dos los casos en las que ha tenido la ocasión de pronunciarse, adoptando, sin embargo, soluciones contradictorias. La primera vez, con ocasión del asunto Delfi c. Estonia ${ }^{39}$ en el 2015, el TEDH refrendó la decisión de las autoridades nacionales de sancionar a una compañía por los daños ocasionados a un particular, al permitir la circulación de amenazas y discursos de odio en la red. En una segunda ocasión, en 2016, como veremos más adelante, eximió de toda responsabilidad a la empresa.

Delfi S.A. era una compañía titular de uno de los portales de noticias de Internet más grandes de Estonia. El 24 de enero de 2006 publicó en el portal una nota crítica acerca de las actividades comerciales desarrolladas por una compañía de ferry, sucediéndose a continuación un conjunto de amenazas contra su accionista mayoritario. Los abogados de este último solicitaron la retirada de dichos comentarios, así como una compensación por daños morales; pretensión esta última que sería rechazada por la compañía, razón por la que terminaría siendo condenada por los tribunales. En este asunto el TEDH entendió que la decisión del tribunal nacional entraba dentro de los supuestos aceptados por el CEDH y desestimó la pretensión del recurrente. El criterio decisivo para los jueces de Estrasburgo fue que el portal de internet tenía una gran infraestructura, ejercía una actividad lucrativa y no había previsto medios para remover de forma inmediata los comentarios y noticias potencialmente lesivas contra terceros ${ }^{40}$.

\footnotetext{
en.asp?fileid $=23456 \&$ lang $=$ en

${ }^{36}$ STEDH Castells contra España, de 23 de abril de 1992 [par. 42] y Fuentes Bobo contra España, de 29 de febrero de 2000 [par. 43]

${ }^{37}$ Sobre este tema, véase el interesante trabajo de Manuel Fernández Salmerón, "Digitalización y convergencia multimedia. Desafíos jurídicos de la comunicación social ante el avance tecnológico", [en Lorenzo Cotino Hueso, coord.: Libertad en Internet: la red y las libertades de expresión e información Tirant lo Blanch, Valencia, 2007], pp. 235-320.

38 App n ${ }^{\circ}$. 3111/10. Case of Ahmet Yildirim v. Turkey, disponible en http://hudoc.echr.coe.int/ eng? $\mathrm{i}=001-139068$

${ }_{39}$ Delfi A.S. c. Estonia [GC], nº $64569 / 09$, ECHR, 2015, disponible en http://hudoc.echr.coe.int/ sites/fra/pages/search.aspx?i=001-126635

${ }^{40}$ Para más información sobre este caso puede consultarse Mart Susi, "Defli AS v. Estonia", en
} 
En una segunda ocasión en la que el TEDH tuvo la oportunidad de pronunciarse (caso Magyar Helsinki Bizottság c. Hungría, de 8 de noviembre de 2016) adoptó una posición totalmente contraria, eximiendo a la empresa que gestiona el portal de internet de cualquier responsabilidad. Así, el 2 de febrero de 2016, el tribunal consideró en su sentencia $n^{\circ}$. 22947/13 que el órgano de autorregulación de contenidos de internet húngaro (Magyar Tartalomszolgáltatók Egyesülete, MTE) y el portal de noticias de internet Index.hu Zrt c., no eran responsables de los comentarios ofensivos publicados por los lectores en sus respectivas webs, revocando así la decisión condenatoria de la Corte húngara. En este caso el tribunal tuvo en cuenta como criterio para determinar la existencia de responsabilidad, la capacidad de control y gestión de la empresa (se trataba en este caso de un portal gestionado por una modesta asociación sin ánimo de lucro) ${ }^{41}$ y el potencial lesivo de los mensajes que circulaban en la red (no se trataba de mensajes potencialmente peligrosos ni incitadores a la violencia, sino de expresiones vulgares de uso corriente), lo que reducía el impacto atribuido a los mensajes ${ }^{42}$.

En definitiva, de lo expuesto en estos casos podría afirmarse que para el TEDH puede existir una culpa in vigilando de los portales en internet ante los comentarios de usuarios aun sin haber recibido reclamación previa al respecto. Dicha responsabilidad, sin embargo, no parece ser automática ni inmediata, sino que dependerá de las circunstancias de cada caso.

\subsection{Reflexiones críticas en torno a la autorregulación en el marco de la Unión Europea}

La primera referencia al discurso de la incitación al odio en internet en el marco de la UE la encontramos en la Directiva 2000/31/CE, de 8 de junio de 2000, denominada "Directiva sobre el comercio electrónico", cuyo artículo 3 prevé la posibilidad de interrumpir el servicio por "los Estados miembros, siempre que ello tenga por finalidad luchar contra la incitación

American Journal of International Law, 2 (2014), pp. 295-302; Víctor Manuel Collí Ek, “¿Libertad de expresión o responsabilidad? ¿Cuándo, en el caso de portales sobre noticias, de acuerdo con la Corte Europea de Derechos Humanos? Análisis y lecturas actuales”, en Revista Mexicana de Derecho Constitucional, 33 (2015), pp. 195-210 y Carlotta Maccioni "Diffamazione online: quando gli Internet Service Provider rispondono per i contenuti diffamatori pubblicati da terzi?", en https://medium.com/ argomenti-di-diritto-dei-media-digitali/diffamazione-online-quando-gli-internet-service-providerrispondono-per-i-contenuti-diffamatori-3221756f93f0

41 Mario Hernández Ramos, "El derecho de acceso a la información pública en la jurisprudencia del TEDH: un derecho instrumental imprescindible para la eficacia del Convenio desde la teoría general de los derechos", en Teoría y Realidad Constitucional, 42 (2018), pp. 483-509.

42 Para más información véase Carlotta Maccioni, "Diffamazione online: quando gli Internet Service Provider rispondono per i contenuti diffamatori pubblicati da terzi?", en https://medium.com/ argomenti-di-diritto-dei-media-digitali/diffamazione-online-quando-gli-internet-service-providerrispondono-per-i-contenuti-diffamatori-3221756f93f0

Araucaria. Revista Iberoamericana de Filosofia, Política, Humanidades y Relaciones Internacionales, año $22, \mathrm{n}^{\circ} 45$. Tercer cuatrimestre de 2020. Pp. 291-310. ISSN 1575-6823 e-ISSN 2340-2199 https://dx.doi.org/10.12795/araucaria.2020.i45.12 
al odio por motivos de raza, sexo, religión o nacionalidad, así como las violaciones de la dignidad humana de personas individuales". Esta Directiva abre la posibilidad de exigir a las empresas el principio de "diligencia debida", recogiendo así la tendencia general de delimitar la responsabilidad de las plataformas intermediarias según el principio de "conocimiento efectivo". Por esta razón, conforme a esta disposición, se entiende que, a priori, estas plataformas no tienen la obligación de detectar los contenidos ilícitos, siendo responsables únicamente en los casos en los que se tenga un conocimiento efectivo de los contenidos alojados en su web. El problema radica en saber qué tipo de notificación debe entenderse suficiente para presumir que la empresa intermediaria está informada del contenido que circula en la web, derivándose de su inacción una responsabilidad por negligencia.

A nuestro juicio esta solución no deja de ser cuestionable en nuestros días. No podemos olvidar que esta directiva data del año 2000, año en el que Google comenzó a operar en Europa. Desde entonces el escenario digital ha cambiado radicalmente. Además, atendiendo a la reciente jurisprudencia del TEDH, no puede descartarse la idea de que proveedores de servicios de internet tengan que asumir responsabilidades por omisión de los contenidos alojados en la web $^{43}$, incluso aun sin tomar parte en la creación de la información que circula en la misma.

El compromiso de la UE contra el discurso de odio on line ha sido claro, como se deduce de la intervención del primer Vice-Presidente de la Comisión Europea, Timmerman, en la Comisión de Libertades Civiles, Justicia y Asuntos de Interior con ocasión de una audiencia sobre antisemitismo, islamofobia y discurso de odio en el Parlamento Europeo el 29 de junio de 2015, la cual afirmó:

I think we need to look at the new areas where hate speech is propagated, which is mainly on the Internet. And the Commission takes that very seriously indeed. And we will need to look for strategies to tackle the issue. Things that people will not say in the public sphere when they meet other people, they will easily propagate on the Internet. They will easily say the most horrible things about other people on the Internet. And it gets a life of its own. There is a whole mythology surrounding the age-old stories of Jews being detached from the countries where they live in because they have some sort of cosmopolitan idea of doing away with national interest, etc. This stuff that has been going on for centuries is back, and it is big again on the Internet ${ }^{44}$.

${ }^{43}$ Iñigo de la Maza-Gazmuri, Responsabilidad de los proveedores de servicios de internet por infracción de los derechos de autor, pp. 33-64, http://www.udp.cl/descargas/facultades_carreras/ derecho/pdf/investigaciones/Cuadernos_de_analisis_Coleccion_Derecho_Privado/numero1/

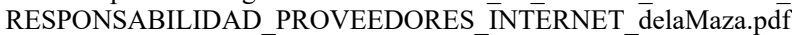

${ }^{44}$ Committee of the European Parliament on Civil Liberties, Justice and Home Affairs, hearing on Anti-Semitism, Islamophobia and hate speech http:/ec.europa.eu/commission/2014- 2019/ timmermans/announcements/transcript-first-vice-president-timmermans-closing-remarks- 
Siguiendo esta línea, el Consejo de la UE publicó el 27 de junio del 2016 las "Directrices de la UE sobre derechos humanos relativas a la libertad de expresión en Internet y fuera de Internet", que incorporan por primera vez la idea de que los derechos humanos también deben ser protegidos en la red. Estas directrices destacan la importancia de generar confianza en este medio para que pueda alcanzar su potencial como facilitador del desarrollo y de la innovación, especialmente en lo que respecta a la libertad de expresión. Precisamente, para hacer posible esta pretensión, desde el Parlamento europeo se propuso una autorregulación en los años noventa que, con el tiempo, se materializaría en la adopción de un código de conducta. De este modo, el 25 de enero de 1999, el Parlamento Europeo aprobó por Decisión no . 276/1999/CE un plan plurianual de acción comunitaria para propiciar una mayor seguridad en la utilización de internet mediante la lucha contra los contenidos ilícitos y nocivos en las redes mundiales en la que se reconoce expresamente (artículo 3), la obligación de

(...) fomentar la autorregulación del sector y los mecanismos de supervisión de los contenidos (por ejemplo, los relativos a contenidos tales como la pornografía infantil o aquellos que inciten al odio por motivos de raza, sexo, religión, nacionalidad $\mathrm{u}$ origen étnico) $(\ldots)^{45}$.

Todo esto explica que la Comisión europea y cuatro grandes empresas del sector de las TIC (YouTube, Twitter, Facebook, Microsoft) hayan adoptado el "Código de conducta para combatir la incitación ilegal al odio en Internet en línea", asumiendo, entre otros compromisos, el establecimiento de filtros de detección de contenidos lesivos (o potencialmente lesivos) y su retirada en un plazo de 24 horas. En esta línea, se ha invitado a los Estados miembros y a los prestadores de servicios a adoptar medidas eficaces, apropiadas y proporcionadas para combatir los contenidos ilícitos en línea ${ }^{46}$. Ahora bien, más allá de las medidas adoptadas y las buenas intenciones que se presume a la autorregulación, no podemos dejar de criticar esta solución por varias razones. Por una parte, el citado código de conducta implica, en la práctica, una privatización de las funciones estatales, ya que son empresas privadas y no autoridades públicas, las que, determinan lo que es lícito o no en la red. Asimismo, siguiendo lo dispuesto en el código de conducta, serán estas empresas (actores privados como Twitter, YouTube...) las que, sin previa intervención judicial, removerán los mensajes que consideren potencialmente lesivos, quedando así las leyes degradadas a un papel secundario y reemplazadas por los términos del servicio de los diferentes servidores. Circunstancia ésta que no deja de ser

duringcommittee-civil-liberties-justice_en

${ }^{45}$ Diario Oficial $\mathrm{n}^{\circ}$. L 033 de 06/02/1999.

${ }^{46}$ El 1 de marzo de 2018 se publicó una Recomendación 2018/334 de la Comisión sobre medidas para combatir eficazmente los contenidos ilícitos en línea.

Araucaria. Revista Iberoamericana de Filosofía, Política, Humanidades y Relaciones Internacionales, año $22, \mathrm{n}^{\circ} 45$. Tercer cuatrimestre de 2020. Pp. 291-310. ISSN 1575-6823 e-ISSN 2340-2199 https://dx.doi.org/10.12795/araucaria.2020.i45.12 
contradictoria con lo dispuesto en el articulo 52 de la CDFUE, cuyo apartado 1 afirma que

\begin{abstract}
cualquier limitación del ejercicio de los derechos y libertades reconocidos por la presente Carta deberá ser establecida por la ley y respetar el contenido esencial de dichos derechos y libertades. Sólo se podrán introducir limitaciones, respetando el principio de proporcionalidad, cuando sean necesarias y respondan efectivamente a objetivos de interés general reconocidos por la Unión o a la necesidad de protección de los derechos y libertades de los demás ${ }^{47}$.
\end{abstract}

A pesar de que, según datos de la Comisión europea la autorregulación ha dado un buen resultado en la lucha contra la incitación al odio on line, insistimos en que esta medida no deja de ser cuestionable. Y es que, con ella no se resuelve el problema de raíz, sino que se garantiza únicamente una "limpieza" de los mensajes y contenidos violentos en internet por parte de actores privados (empresas TIC) de una forma discrecional y poco controlada. Además, esta solución puede dar lugar a perjuicios para los usuarios, que verán más o menos limitados sus derechos en función de las políticas de control de los diferentes operadores de la red. Llama la atención la gran diferencia existente entre esta solución y la del sistema norteamericano. Piénsese, en este sentido, que el art. 230 del Communication Decency Act (sec. c, 1) de los EEUU excluye categóricamente al proveedor de los servicios interactivos digitales de cualquier responsabilidad, de la misma manera que podría serlo un editor (publisher) de los contenidos informativos producidos por otros. Precisamente por este motivo, los tribunales (sirva de ejemplo la decisión de la Corte del Northern District of California, 18 de noviembre de 2016, Fields $v$. Twitter) han rechazado las acciones presentadas por familiares de víctimas de un atentado terrorista islámico contra Twitter y otros socialnetwork, acusados de haber permitido la apertura de cuentas en sus portales a través de las cuales los seguidores de ISIS hacían propaganda de su ideario extremista. Para los jueces de la Corte de los EEUU, el gestor de la plataforma de social networking no puede, en ningún caso, ser considerado responsable de los contenidos producidos y difundidos por los usuarios del servicio.

\title{
Conclusiones
}

La libertad de expresión constituye uno de los fundamentos esenciales de una sociedad democrática y una de las condiciones primordiales de su progreso y desarrollo, razón por la que tiene que ser protegida y garantizada por los poderes públicos. Ahora bien, los límites entre las simples críticas y aquellas

${ }^{47}$ Diario Oficial ${ }^{\circ}$. C 364/1 de 18 de diciembre de 2000. 
expresiones que constituyen discurso de odio son, en ocasiones, difíciles de definir. A esta circunstancia contribuye el hecho de que no exista un consenso general sobre lo que debe entenderse por discurso de odio, expresión esta última fuertemente condicionada por la experiencia histórica de cada cultura y conformada por las circunstancias sociales, económicas y religiosas e incluso las políticas de cada sociedad. De hecho, podría afirmarse que la conciencia colectiva ante las conductas de odio surge a partir de la Segunda Guerra Mundial, evolucionando como respuesta frente a planteamientos políticos excluyentes y totalitarios (fascismo, antisemitismo...). Posteriormente se puso de manifiesto por motivos racistas o de segregación racial, para concretarse en torno a conflictos étnicos o diversas formas de discriminación como la homofobia, la transfobia, la xenofobia, la intolerancia religiosa e, incluso, más recientemente la romafobia, la aporafobia o la gerontofobia ${ }^{48}$.

La dificultad radica pues, en alcanzar un equilibrio entre la oportunidad de regular el ejercicio de la libertad de expresión para evitar su uso abusivo y la necesidad de garantizar su protección como derecho fundamental. Por lo que a Europa se refiere, puede afirmase que existe una mayor aceptación de la limitación del derecho a libertad de expresión que en la tradición jurídica norteamericana (EEUU), en la que, a priori, se rechaza cualquier injerencia de los poderes públicos y en donde se entiende que ésta debe difundirse en el free Marketplace of ideas sin tutelas externas. De hecho, en el marco del Consejo de Europa la limitación de la libertad de expresión es posible ante acciones que no conllevan per se una apelación a la comisión de un delito, a una acción hostil, discriminatoria o violenta contra un grupo social, como es el caso de la promoción o justificación del odio.

Si bien la incitación al odio siempre ha existido y se ha manifestado a través de medios tradicionales (prensa, TV, radio...), no cabe ninguna duda de que hoy, tras la irrupción de internet en nuestras vidas, ésta también se encuentra en el ciberespacio, difundiéndose con rapidez y a gran escala. De hecho, podría afirmarse que el potencial lesivo de estos discursos aumenta en el ciberespacio por sus propias características intrínsecas: anonimato, ausencia de control centralizado, ilimitación de espacio y tiempo...

Por lo que al continente europeo se refiere, en los últimos años se ha ido desarrollando un basto arsenal normativo y jurisprudencial (tanto en el marco del Consejo de Europa, como de la UE), con el fin de contrarrestar la incitación al odio en el ciberespacio y determinar su responsabilidad por los daños causados. En este contexto los portales de internet han tomado un gran protagonismo. Así, por ejemplo, a partir de la jurisprudencia del TEDH podría afirmarse que, en determinadas circunstancias, existe una culpa in vigilando de los portales

\footnotetext{
${ }^{48}$ Parte de esta información ha sido extraída de la Circular 7/2019, sobre pautas para interpretar los delitos de odio tipificados en el art. 510 CP. BOE, nº 124, de 24 de mayo de 2019.
} 
en internet ante lesiones o daños causados por perjuicios a terceros. Para el tribunal la responsabilidad de los portales se determinará atendiendo a cada caso concreto, en función de la dimensión del portal, el carácter profesional de su gestión y la existencia de la actividad comercial. Solución ésta, bien distinta a la que se aplica en el sistema norteamericano.

Por otra parte, dicho protagonismo también se constata en el marco de la UE, en el que grandes proveedores como Facebook, Twitter y Google han asumido una serie de compromisos (autorregulación en el marco de un Código de conducta) relativos al establecimiento de filtros de detección de contenidos y retirada de la red de mensajes lesivos. Circunstancia ésta que, si bien puede ser una buena solución política, no deja de ser criticable desde el punto de vista jurídico: se ha dejado en manos de operadores privados (de tradición cultural exógena a la europea) el control de un derecho fundamental. Solución ésta que, a todas luces, es contraria a la CDFUE, cuyo artículo 52, exige que cualquier limitación del ejercicio de los derechos y libertades sea establecida por la ley. 


\section{Referencias bibliográficas:}

Abbate, Janet, Inventing the Internet, Cambridge, MIT Press, 1999, pp. 36-111. Allport, Gordon W., The nature of prejudice, Addison-Wesley Publishing company, London, 1954.

Balkin, Jack M. "Old-school/new-school speech regulation", en Harvard Law Review, 127 (2014), pp. 2296- 2342.

Benkler, Yochai, La ricchezza della rete, Milano,Università Bocconi Editore, 2007.

Calvès, Gwénaëlle, "Les discours de haine et les normes internationales", Esprit, 10 (2015), pp. 56-66.

Carrea, Simone, "The ECHR in the cyberspace: does the power to infringe always entail the duty to protect?", en Diritti umani e diritti internazionale, 13 (2019), pp. 133-155.

Collí Ek, Víctor Manuel, “¿Libertad de expresión o responsabilidad? ¿Cuándo, en el caso de portales sobre noticias, de acuerdo con la Corte Europea de Derechos Humanos? Análisis y lecturas actuales", en Revista Mexicana de Derecho Constitucional, 33 (2015), pp. 195-210.

Cruz Ángeles, Jonatán, "Libertad de expresión versus discurso de odio en internet: análisis del marco jurídico internacional", [en Blanca Martín Ríos, coord.: La prevención y represión del discurso del odio: hacia la construcción multidisciplinar de la tolerancia, Madrid, Aranzadi Thomson Reuters, 2019], pp. 211-240.

Frost, Bryan-Paul \& Sikkenga, Jeffrey, History of American Political Thought. Maryland, Lexington, 2019.

Girard, Charles, "Pourquoi punir les discours de haine ?", en Esprit, 10 (2015), pp. 11-22.

Hernández Ramos, Mario "El derecho de acceso a la información pública en la jurisprudencia del TEDH: un derecho instrumental imprescindible para la eficacia del Convenio desde la teoría general de los derechos", en Teoría y Realidad Constitucional, 42 (2018), pp. 483-509.

Leets, Laura, "Responses to Internet Hate Sites: Is Speech Too Free in Cyberspace?", Communication Law and Policy, 6 (2001), pp. 287-317.

Maccioni, Carlotta "Diffamazione online: quando gli Internet Service Provider rispondono per i contenuti diffamatori pubblicati da terzi?", en https://medium.com/argomenti-di-diritto-dei-media-digitali/diffamazioneonline-quando-gli-internet-service-provider-rispondono-per-i-contenutidiffamatori-3221756f93f0 
Maza-Gazmuri, Iñigo de la, Responsabilidad de los proveedores de servicios de internet por infracción de los derechos de autor, pp. 33-64, http:// www.udp.cl/descargas/facultades_carreras/ derecho/pdf/investigaciones/ Cuadernos_de_analisis_Coleccion_Derecho_Privado/numero1/ RESPONSABILIDAD_PROVEEDORES_INTERNETETdelaMaza.pdf

McGonagle, Tarlach / Donders, Yvonne, The United Nations and Freedom of Expression and Information, Cambridge University Press, Cambridge, 2015.

Menzi, Maurizio / Falleta, Pietro, Il diritto del Web. Casi e materiali, Trento, CEDAM, 2015.

Moya García, Rodrigo, "La Libertad de Expresión en la Red Internet", en Revista Chilena de Derecho Informático, 2 (2003), pp. 89-108.

Nicollier, Pascal, La Déclaration des Droits de l'Homme et Citoyen du 26 août 1789, Fribourg, 1995.

Oyediran, Joanna, "Article 13(5) of the American Convention on Human Rights", [en Sandra Coliver, coord.: Striking a balance. Hate Speech, Freedom of Expression and Non-discrimination, University of Essex, London, 1992], pp. 33-35.

Rodríguez Vázquez, Daniel "El genocidio de Ruanda: análisis de los factores que influyeron en el conflicto", en Instituto Español de Estudios Estratégicos, Documento de opinión 59 (2017)

Rollnert Liern, Göran, "El discurso del odio: una lectura crítica de la regulación internacional", en Revista Española de Derecho Constitucional, 115 (2019), pp. 81-109.

Susi, Mart, "Delfi AS v. Estonia", en American Journal of International Law, 2 (2014), pp. 295-302. 